\title{
Observation of supersymmetric scattering in photonic lattices
}

\author{
Matthias Heinrich, ${ }^{1,2, *}$ Mohammad-Ali Miri, ${ }^{2}$ Simon Stützer, ${ }^{1}$ Stefan Nolte, ${ }^{1}$ \\ Demetrios N. Christodoulides, ${ }^{2}$ and Alexander Szameit ${ }^{1}$ \\ ${ }^{1}$ Institute of Applied Physics, Abbe Center of Photonics, Friedrich-Schiller-Universität Jena, Max-Wien-Platz 1, o7743 Jena, Germany \\ ${ }^{2}$ CREOL, The College of Optics and Photonics, University of Central Florida, 4304 Scorpius St., Orlando, Florida 32816, USA \\ ${ }^{*}$ Corresponding author: matthias.heinrich@uni-jena.de
}

Received August 4, 2014; revised September 23, 2014; accepted September 25, 2014; posted September 26, 2014 (Doc. ID 220367); published October 17, 2014

Supersymmetric (SUSY) optical structures display a number of intriguing properties that can lead to a variety of potential applications, ranging from perfect global phase matching to highly efficient mode conversion and novel multiplexing schemes. Here, we experimentally investigate the scattering characteristics of SUSY photonic lattices. We directly observe the light dynamics in such systems and compare the reflection/transmission properties of SUSY partner structures. In doing so, we demonstrate that discrete settings constitute a promising testbed for studying the different facets of optical supersymmetry. (c) 2014 Optical Society of America

OCIS codes: (290.0290) Scattering; (230.7370) Waveguides; (260.2710) Inhomogeneous optical media. http://dx.doi.org/10.1364/OL.39.006130

The concept of supersymmetry (SUSY) was originally developed in quantum field theory as a means to unify the mathematical treatment of bosons and fermions [1]. While to this date no evidence for SUSY behavior has been found in nature, the underlying theoretical formalism can be adapted in a variety of different fields of physics, ranging from nonrelativistic quantum mechanics [2] to the design of semiconductor heterostructures [3] and the optimization of frequency conversion in quantum cascade lasers, to name a few [4]. Recently, optical SUSY was found to provide a systematic approach to phase matching and mode manipulation in wave-guiding structures [5-7]. Along these lines, supersymmetric mode converters have been proposed as versatile building blocks for readily scalable, highly efficient, and fully integrated mode division multiplexing arrangements [].

Yet, the consequences of SUSY are by no means limited to guided waves. The very principles, which serve to establish perfect global phase matching between waveguides linked by supersymmetric transformations, can also have a profound impact on the scattering characteristics. As it turns out, two SUSY partner structures exhibit the same intensity reflection and transmission coefficients for all angles of incidence. This peculiar behavior could render two apparently dissimilar structures virtually indistinguishable, suppress reflections at their interface, or make

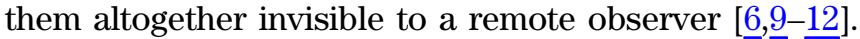
Based on these considerations, it is even possible to supersymmetrically extend transformation optics to enable the design of low-index dielectric equivalent structures that faithfully mimic the optical properties of high-contrast, or negative-index, arrangements [13].

In this work, we experimentally investigate and report the first observation of light scattering from SUSY structures. Supersymmetric arrangements are realized in coupled networks of photonic lattices fabricated via femtosecond laser writing schemes [14]. The corresponding light dynamics in such systems are directly visualized by means of waveguide fluorescence microscopy [15]. Our findings extend the scope of SUSY experiments beyond the previously investigated bound state scenarios []], and in doing so, illustrate how discrete arrangements readily allow for the implementation and observation of scattering-invariant transformations mediated by optical SUSY.

In optics, supersymmetric transformations involve a factorization of the Hamiltonian-like operator $\mathcal{H}=$ $-\partial_{x}^{2}-k_{0}^{2} n^{2}(x)$, which governs the propagation of light in a continuous refractive index landscape $n(x)$ in the $x-z$ plane [5]. Here, $k_{0}$ represents the vacuum wave number. For a Hermitian conjugate pair of operators $\mathcal{A}$ and $\mathcal{A}^{\dagger}$, one can readily see that the Hamiltonian $\mathcal{H}^{(1)}=\mathcal{A}^{\dagger} \mathcal{A}$ shares a common set of propagation constants, or eigenvalues $\lambda$, with $\mathcal{H}^{(2)}=\mathcal{A A}^{\dagger}$. Moreover, it is known that for any incident angle $\theta$, the complex reflection and transmission coefficients $r^{(1,2)}$ and $t^{(1,2)}$ associated with the corresponding index landscapes $n^{(1,2)}$ are equal to up a phase [5]. In terms of intensity, the respective reflectivities $R^{(\overline{1})}=R^{(2)}=$ $\left|r^{(1,2)}\right|^{2}$ and transmittivities $T^{(1)}=T^{(2)}=\left|t^{(1,2)}\right|^{2}$ of these two structures are strictly identical.

The conceptual framework of SUSY can be naturally extended to discrete systems such as periodic arrays of evanescently coupled waveguides, or "photonic lattices." In the coupled-mode approximation, the evolution of wave packets in this latter type of arrangements is described by a discrete Hamiltonian,

$$
-i \partial_{z} A=H A .
$$

Here, $A=\left(a_{1}, \ldots, a_{n}\right)^{T}$ is the state vector comprised of the individual waveguide amplitudes $a_{n}$, and the symmetric square matrix $H=\left(\delta_{m-1, n}+\delta_{m+1, n}\right) C_{n}+\delta_{m, n} \beta_{n}$ contains the individual propagation constants $\beta_{n}$ of the lattice sites on its main diagonal, and the nearest neighbor coupling coefficients $C_{n} \equiv C_{n, n+1}=C_{n+1, n}$ in the secondary diagonals [16].

In contrast to the continuous regime [5], the discrete Hamiltonian of a photonic lattice can be factorized, in a systematic and efficient manner, by a number of exact algebraic techniques [17]. Here we employ the so-called QR-method to find an orthogonal matrix $Q$ and an upper triangular matrix $R$, such that $H=H^{(1)}-\lambda_{k}=Q R$. In 
subtracting its corresponding eigenvalue $\lambda_{k}$, any state can thus be eliminated from the partner Hamiltonian $H^{(2)}=R Q+\lambda_{k}$. Note that the ability to address arbitrary states, regardless of their position within the spectrum, sets the QR factorization apart from the Cholesky method [17], which is reminiscent of the continuousindex approach, and is only valid for positive-definite matrices, i.e., the removal of the fundamental mode.

Having established discrete optical SUSY, the question naturally arises as to how scattering phenomena can be described in such guided-wave arrangements, without violating the underlying coupled-mode theory. To do so, we here consider only scattering processes within the transmission bands of the lattice structures as established through the tight-binding model. Along these lines, this nearest-neighbor coupling can lead to transverse transport, diffraction, and even reflection/transmission of waves at inhomogeneities in extended lattices [18-21]. In particular, owing to the periodic relation between transverse momentum and propagation constant (in the propagation band), wave packets occupying the vicinity of the diffraction relation's inflection points are known to propagate without significant broadening [18]. In our experiments, we will use this particular regime to probe the scattering properties of SUSY photonic lattices.

Note that, strictly speaking, SUSY is a global system property. Appending any number of waveguides to either side of a finite array invariably distorts the eigenvalue spectrum. As a consequence, one cannot simply immerse two SUSY partner structures in identical homogeneous background lattices to provide an interface for scattering experiments. Instead, the construction has to be based on the entire fundamental system, including the uniform side regions. The central domain then constitutes a defect, capable of supporting localized modes. Figure 1(a)

shows such a defect domain comprised of four identical waveguides, when their propagation constants have been elevated by $1.5 \times$ the coupling coefficient $C_{0}$ of the background lattice: $\Delta \beta=1.5 C_{0}$. The corresponding modes for an overall system stretching 18 waveguides on either side of the defect, i.e., 40 lattice sites in total, are shown in Fig. 1(b). Most of their respective eigenvalues $\lambda_{j}$ fall within the propagation band $\pm 2 C_{0}$. The only exceptions are two localized states residing in the semi-infinite gap above the band. SUSY transformations can now be employed to eliminate specific bound states. The resulting partner system is largely similar to the fundamental one, as deviations from the background lattice are confined to the area formerly occupied by the now absent mode. Figure 1(c) displays the structure of the partner lattice obtained by QR factorization. Note that the SUSY transformation yields joint modifications in both the propagation constants and coupling coefficients, and leads to an asymmetric lattice. Yet, SUSY does not introduce a sense of directionality: Reversing the direction of the transverse coordinate would yield an equally valid superpartner. Figure $1(d)$ shows the corresponding lattice modes, including the single localized defect state. While the density of states in the propagation band depends on the extent of the surrounding lattice, the actual wave dynamics in the vicinity of the defect domain remain unaffected by such boundary effects. In that sense, the background lattice provides a continuum of propagating waves, and can be truncated (or expanded) at will, as was done here to maintain the overall system size of 40 waveguides. Similarly, the QR factorization algorithm allows for the removal of localized states residing on negatively detuned defect domains, even though their eigenvalues lie within the Bragg gap of the surrounding lattice [see Figs. $\underline{1(\mathrm{e})}-\underline{1(\mathrm{~h})}]$.

(e) Fundamental system
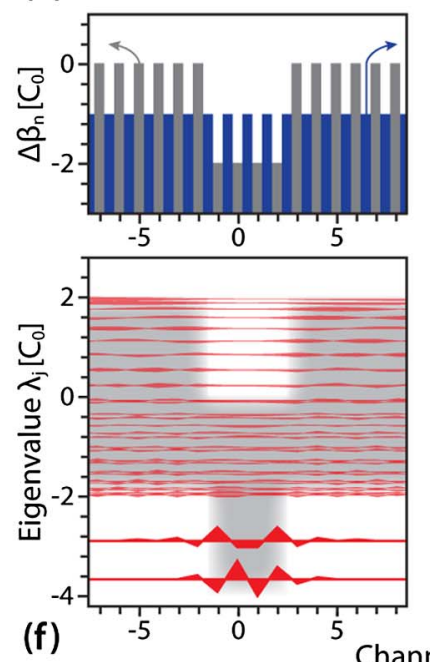
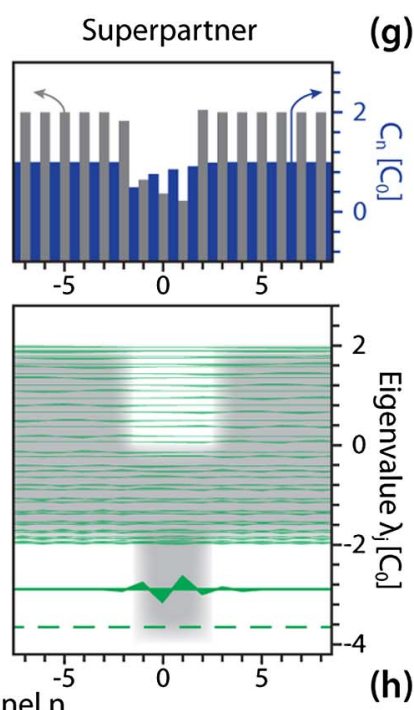

(b)
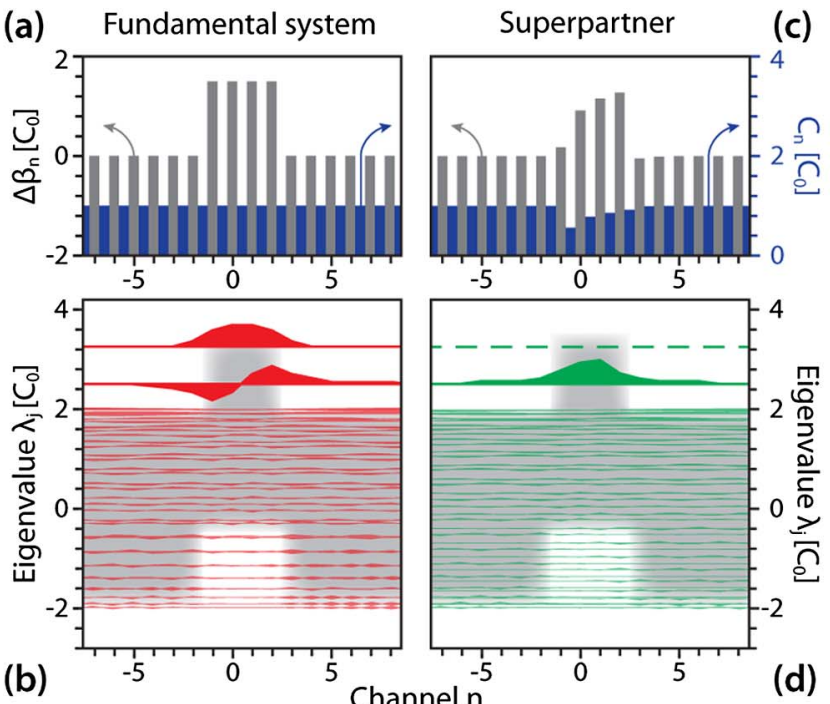

(d)

Fig. 1. (a) Positively detuned defect domain $\left(\Delta \beta=1.5 C_{0}\right)$ embedded within a homogeneous background lattice. Shown are the propagation constants $\beta_{n}$ of the individual lattice sites (gray bars, left scale), and the coupling coefficients $C_{n}$ between them (blue bars, right scale). (b) Lattice modes for the case of 18 waveguides on either side of the defect, i.e., 40 lattice sites in total. The mode profiles of the defect and radiation states are depicted as a function of the waveguide numbers, while the vertical position of each mode indicates the respective eigenvalue $\lambda_{j}$. The defect domain supports two bound states residing above the propagation band. (c) Superpartner lattice obtained by removing the fundamental defect state via $\mathrm{QR}$ factorization, and (d) its single bound mode residing above the band. (e) Negatively detuned defect domain $\left(\Delta \beta=-2 C_{0}\right)$ embedded within a homogeneous background lattice, and (f) its lattice modes. The defect domain supports two bound states residing below the propagation band. (g) Superpartner lattice obtained by removing the lowest defect state via QR factorization, and (h) its single bound mode residing below the band. 
(a)
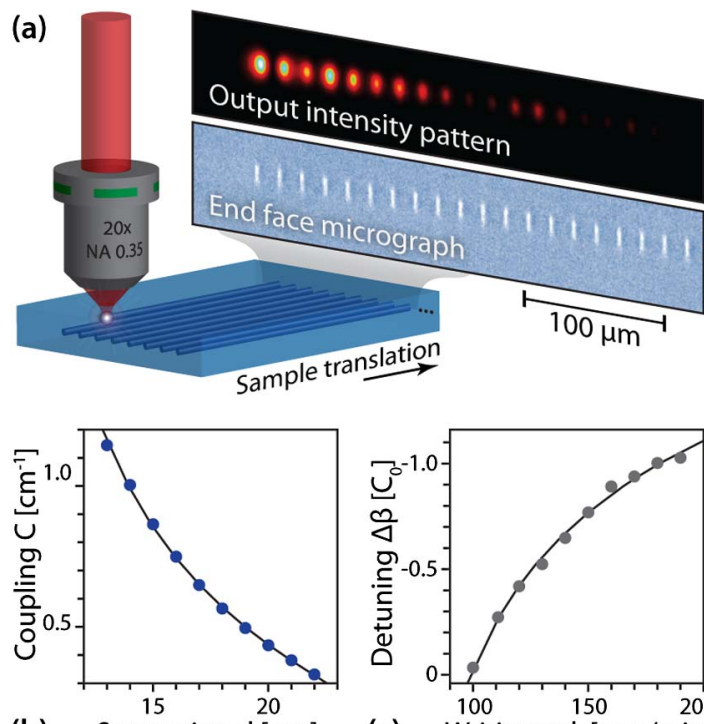

(b) Separation d [ $\mathrm{mm}]$

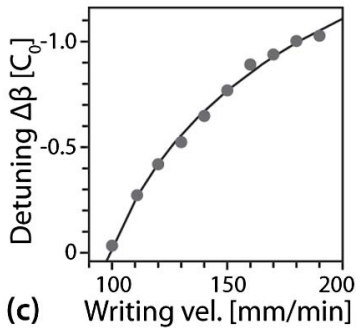

(c) Writing vel. [mm/min]

Fig. 2. (a) Schematic of the waveguide inscription method. The inserts show a micrograph of the sample end face, and a typical output intensity pattern observed at $633 \mathrm{~nm}$. (b) Dependence of the coupling coefficient $C$ on the waveguide separation $d$. (c) Influence of the writing velocity on the detuning $\Delta \beta$ of a waveguide with respect to a reference guide inscribed at $100 \mathrm{~mm} / \mathrm{min}$.

To experimentally study the scattering properties of discrete supersymmetric arrangements, we employed the femtosecond laser direct writing technique [14] [see Fig. 2(a). By choosing appropriate writing velocities and waveguide spacings, photonic lattices with the desired propagation constants and coupling coefficients [see Figs. 2(b) and 2(c)] were inscribed in $10 \mathrm{~cm}$ long fused silica samples. A confined, diffraction-free probe beam was generated by placing the weakly focused excitation beam (wavelength $633 \mathrm{~nm}$ ) in the background lattice, and slightly tilting the sample by an angle corresponding to the inflection point of the lattice band [18]. By virtue of the fluorescent properties of our waveguides [15], we directly observed the evolution of the beam and its interaction dynamics with the defect regions. To allow for a quantitative comparison between the observed propagation patterns (irrespective of the different waveguide positions in the superpartner lattices), we numerically extracted the intensity distributions $I_{n}(z)$ of the individual channels. Figure 3 uses this type of intensity plot to highlight the peculiar scattering behavior of SUSY photonic lattices. Shown are the propagation patterns observed in the case of a positively detuned defect domain with $\Delta \beta=C_{0}$ [Intensity $I^{(1)}$, see Fig. 3(a)] and its superpartner [Intensity $I^{(2)}$, see Fig. 3(b)]. In addition to the obvious visual similarity of these two scattering processes, a closer examination reveals a difference between these two intensities $I^{(2)}-I^{(1)}$, a direct outcome of the phase differences involved. Since the incoming wave packet encounters a less abrupt change of lattice parameters in the supersymmetrically deformed defect region, it can penetrate slightly further, resulting in a longer Goos-Hänchen-like displacement along $z$. Nevertheless, both have the same asymptotic shape and carry the same fraction of the overall intensity, as dictated by SUSY. Our
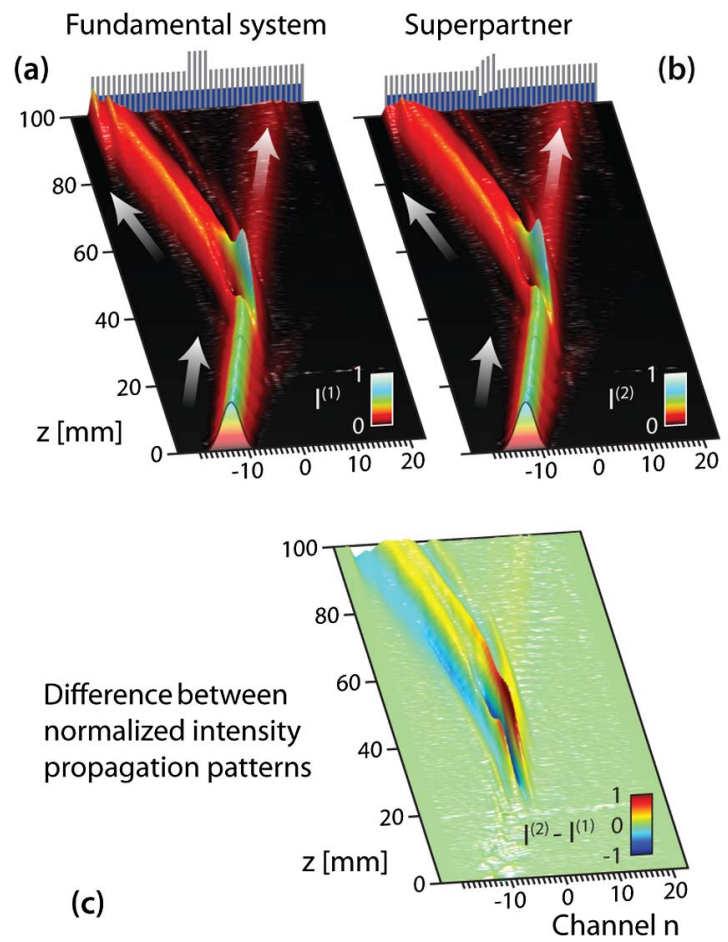

Fig. 3. (a) Observed evolution of a confined wave packet upon partial reflection at a positively detuned defect domain $\left(\Delta \beta=C_{0}\right)$. Plotted is the intensity in the individual lattice sites $n$ along the longitudinal coordinate $z$. (b) Corresponding evolution in the superpartner lattice. (c) Quantitative difference between the patterns arising from a differential GoosHänchen-like shift. The patterns in (a) and (b) are normalized to the same input beam intensity, and the values associated with the color bars are compatible in all subfigures.

experiments clearly demonstrate that the intensity reflection/transmission coefficients of these two partner structures are indeed identical.

In a second set of experiments, we realized a number of different defect arrangements with positive $\left(\Delta \beta=0.5,1.5,2.0,2.5 C_{0}\right)$ as well as negative detunings $\left(\Delta \beta=-2.0,-1.5,-1.0 C_{0}\right)$. Their superpartners were synthesized by removing the respective most strongly localized defect states, i.e., the modes with the highest (lowest) eigenvalue for the positive (negative) defects. We then observed the output intensity distributions at the sample end faces. Compared to the fluorescence method, this measurement is subject to a significantly lower background, and the modal intensities can be extracted with high fidelity to obtain quantitative information about the reflection/transmission behavior. The left side of Fig. 4(a) shows the end face images, normalized with respect to their individual overall intensities. The reflected parts of the input beam occupy the lattice sites with $n \leq-2$, whereas the waveguides $n>2$ carry the transmitted fraction. For the lattice size in our experiments, the reflectivity is therefore given by $R=$ $\sum_{n=-19}^{-2} I_{n} / I_{\text {total }}$. The values of $R$ obtained from the measurements for the defects and their respective superpartners closely match one another within their margins of error. As predicted by the tight binding model [see right side of Fig. 4(a)], the reflectivity increases with the relative strength of the defect. This can be easily 
(a) Fundamental system
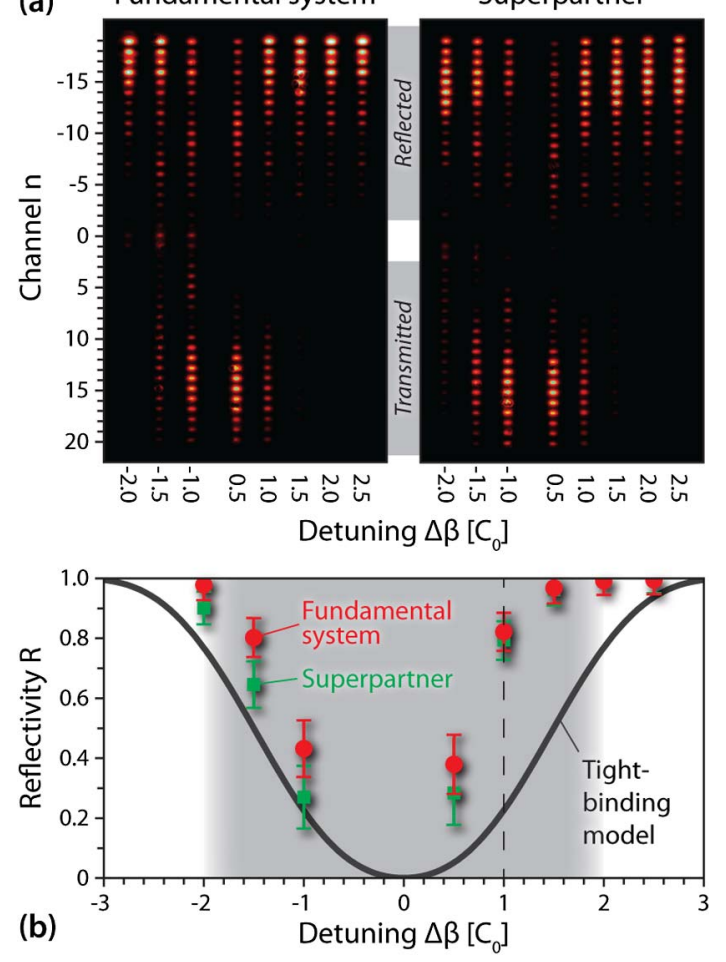

Fig. 4. Reflection/transmission from defect domains and their respective superpartners for different values of detuning. Observed output intensity distributions at $z=100 \mathrm{~mm}$ in the fundamental lattices (left) and SUSY partners (right). The reflected and transmitted parts of the probe beam are located at $n \leq-2$ and $n \geq+3$, respectively. (b) Values of the reflectivity $R$ extracted from the output patterns. For comparison, the theoretical band structure is shown as a solid graph. The dashed vertical line indicates the data points corresponding to the propagation patterns shown in Fig. 3(a)

understood by considering that a sufficiently detuned region can effectively separate the surrounding lattice. If it does not support any states within the propagation band, the defect ideally poses an impenetrable barrier to light contained in the scattered states. As the detuning decreases, so does the fraction of domain modes that lie outside the band. Naturally, at zero detuning, the domain would become indistinguishable from the lattice, and exhibit vanishing reflectivity. Deviations from the theoretical prediction illustrate the fact that the experimental system departs from the ideal tight-binding configuration, e.g., by displaying higher-order couplings [22], which subtly change the shape of the diffraction relation as well as its associated density of states. Nevertheless, lattices related by SUSY transformations clearly resemble one another with respect to their scattering properties. Along these lines, we note that SUSY notions also provide a new, and possibly more intuitive, perspective to previous studies on reflectionless potentials [20,21].

In conclusion, we implemented discrete supersymmetric optical structures in femtosecond laser-written waveguide photonic lattices, and experimentally studied the scattering characteristics of such systems. Our findings constitute the first observation of SUSY scattering behavior in either continuous or discrete systems, and illustrate how lattice-type arrangements readily allow for the implementation and observation of optical SUSY. Of interest will also be an extension of these experiments to non-Hermitian SUSY-synthesized systems, which have recently been predicted to offer particularly unusual scattering behavior $[\underline{23}, \underline{24}]$.

The authors gratefully acknowledge financial support from NSF (grant ECCS-1128520), AFOSR (grants FA9550-12-1-0148 and FA9550-14-1-0037), the German Ministry of Education and Research (Center for Innovation Competence program, grant 03Z1HN31), Thuringian Ministry for Education, Science and Culture (Research group Spacetime, grant no. 11027-514), and the GermanIsraeli Foundation for Scientific Research and Development (grant 1157-127.14/2011). MH was supported by the German National Academy of Sciences Leopoldina (grant LPDS 2012-01).

\section{References}

1. P. Ramond, Phys. Rev. D 3, 2415 (1971).

2. F. Cooper, A. Khare, U. Sukhatme, and A. Khare, Phys. Rep. 251, 267 (1995)

3. J. Radovanović, V. Milanović, Z. Ikonić, and D. Indjin, Phys. Rev. B 59, 5637 (1999).

4. J. Bai and D. S. Citrin, Opt. Express 14, 4043 (2006).

5. M.-A. Miri, M. Heinrich, R. El-Ganainy, and D. N. Christodoulides, Phys. Rev. Lett. 110, 233902 (2013).

6. M.-A. Miri, M. Heinrich, and D. N. Christodoulides, Phys. Rev. A 87, 043819 (2013).

7. H. P. Laba and V. M. Tkachuk, Phys. Rev. A 89, 033826 (2014)

8. M. Heinrich, S. Stützer, M.-A. Miri, R. El-Ganainly, S. Nolte, A. Szameit, and D. N. Christodoulides, Nat. Commun. 5, 3698 (2014).

9. S. Longhi and G. Della Valle, Europhys. Lett. 102, 40008 (2013).

10. S. Longhi, Phys. Rev. A 82, 032111 (2010).

11. S. Longhi and G. Della Valle, Ann. Phys. 334, 3546 (2013).

12. B. Midya, Phys. Rev. A 89, 032116 (2014).

13. M.-A. Miri, M. Heinrich, and D. N. Christodoulides, Optica 1, 89 (2014).

14. A. Szameit and S. Nolte, J. Phys. B 43, 163001 (2010).

15. A. Szameit, F. Dreisow, H. Hartung, S. Nolte, A. Tunnermann, and F. Lederer, Appl. Phys. Lett. 90, 241113 (2007).

16. D. N. Christodoulides, F. Lederer, and Y. Silberberg, Nature 424, 817 (2003).

17. L. Hogben, Handbook of Linear Algebra, (Chapman \& Hall/ CRC, 2006).

18. T. Pertsch, T. Zentgraf, U. Peschel, A. Bräuer, and F. Lederer, Phys. Rev. Lett. 88, 093901 (2002).

19. A. Szameit, H. Trompeter, M. Heinrich, F. Dreisow, U. Peschel, T. Pertsch, S. Nolte, F. Lederer, and A. Tünnermann, New J. Phys. 10, 103020 (2008).

20. A. A. Sukhorukov, Opt. Lett. 35, 989991 (2010).

21. A. Szameit, F. Dreisow, M. Heinrich, S. Nolte, and A. A. Sukhorukov, Phys. Rev. Lett. 106, 193903 (2011).

22. F. Dreisow, A. Szameit, M. Heinrich, T. Pertsch, S. Nolte, and A. Tünnermann, Opt. Lett. 33, 2689 (2008).

23. S. Longhi and G. Della Valle, Phys. Rev. A 89, 052132 (2014).

24. S. Longhi, Opt. Lett. 39, 1697 (2014). 